\title{
Modification of the apoptotic-like effects of MBP protein overexpression in E. coli by fusion with 14-3-3 derived polypeptides
}

\author{
Ewa Markiewicz ${ }^{1,2}$, Grzegorz Wilczynski ${ }^{1}$, Jan Filipski ${ }^{2,3}$ and \\ Jan Szopa ${ }^{1}$ \\ 1 Laboratorium Biochemii Genetycznej, Uniwersytet Wroclawski, Wroclaw, \\ Poland \\ 2 Laboratoire de Mutagénèse, Institut J. Monod, Paris, France \\ ${ }^{3}$ corresponding author: Institut J. Monod, 2 place Jussieu, Tour 43, 75251 , \\ Paris, France. tel: (33-1) 442769 49; fax: (33-1) 442757 16; \\ e-mail: filipski@ijm.jussieu.fr
}

Received 28.08.96; revised 16.11.96; accepted 9.1.97 Edited by P. Cohen

\begin{abstract}
Overexpression of even non-toxic proteins in bacteria causes a starvation-like response: the arrest of bacterial proliferation and apoptotic-like suicidal cell death. We have shown here that, as in the cells of higher organisms, these effects are accompanied by DNA degradation. The fusion with the bacterial MBP of a polypeptide, belonging to the 14-33 family and normally expressed in pumpkin (C. pepo), modifies the apoptotic-like effects of overexpression of this protein in E. coli. Fusion of the full length 14-3-3 protein with the MBP considerably slows down the DNA degradation caused by overexpression of the unmodified MBP. Overexpression of the construct containing a truncated version of the 14-3-3 polypeptide causes immediate arrest of bacterial growth and rapid degradation of the chromosomal DNA. This result suggests that the DNA degradation in bacteria is an active process which can be modified to some extent by an endogenous protein.
\end{abstract}

Keywords: apoptosis, DNA degradation, plant, bacteria, 14-33 protein

Abbreviations: MBP, maltose-binding protein; IPTG, isopropyl- $\beta$-D-thiogalactopyranoside; $\mathrm{kb}$, kilobase (1000 base pairs); SDS, sodium dodecyl sulfate; PAGE, polyacrylamide gel electrophoresis, p-MAL, p-MAL-CDNA ${ }^{14-3-3}$ and p-MalcDNA $^{\Delta N 14-3-3}$ plasmids expressing the MBP or the MBP fused either with the full-size or with the truncated version of 14-3-3 protein, respectively; PKC, protein kinase C

\section{Introduction}

Programmed cell death occurs not only in multicellular organisms but also in bacteria (Yarmolinsky, 1995). It is probably a mechanism which helps bacteria subpopulations to survive in situations of stress. The heterogenity of bacterial populations, caused by induction of mutagenic processes is, in such situations, the rule rather than an exception (Hall, 1990).

Suicidal behaviour is typical of bacteria carrying multicopy plasmids containing a gene under the control of a strong promoter. These bacteria stop growing upon induction of the synthesis of the plasmid-encoded protein. The mechanism of this effect is unclear. One possible explanation is that an unspecific 'protein burden' causes starvation of the bacteria: it has been shown that overexpression of various proteins is accompanied by an uniform growth-rate inhibition (Dong et al, 1995). On the other hand, however, the growth inhibition appears to be stronger than expected (Kurland and Dong, 1996). It is thus possible that upon induction of the synthesis of a gratuitous protein an active apoptotic-like process takes place in the bacterial cell.

In higher organisms, the early steps of apoptosis are accompanied by fragmentation of the chromosomal DNA into large $50-300 \mathrm{~kb}$ long products (Filipski et al, 1990). We wanted to determine whether a similar nucleolytic process occurs in bacteria. As a model system we used the overexpression of polypeptides belonging to the 14-3-3 family. We chose this protein because of its possible involvement in the process of apoptosis in plants (Szopa, 1994).

The abundant, acidic, tissue-specific 14-3-3 protein was initially isolated from bovine brain. Since this discovery several related proteins with different functions have been found in a wide range of organisms including mammals, insects, yeast and plants (for review see Aitken et al, 1992). Recent findings point to a participation of these proteins in the control of the cell cycle and the regulation of gene expression (for reviews see Aitken, 1995; Burbelo and Hall, 1995). Members of the 14-3-3 family activate neurotransmitter synthesis and ADP-ribosylation of proteins, regulate protein kinase $\mathrm{C}$, display a phospholipase $\mathrm{A} 2$ activity and associate with the products of proto-oncogenes, oncogenes and the cdc 25 gene (Reuter et al, 1994; Fu et al, 1994; Morrison, 1994; Conklin et al, 1995). The broad spectrum of activities that are affected suggests that the 14-3-3 proteins have several isoforms and that each isoform has its own unique function. Recently, the cDNA encoding 14-3-3 protein from pumpkin Cucurbita pepo var. patissonina was isolated and sequenced. The nucleotide and deduced amino acid sequences show a very close similarity to known 14-3-3 protein sequences from other sources. The amino acid sequence contains consensus sequences for potential phosphorylation by protein kinase $A$ and $C$ and for calcium-, lipid membrane-, and nucleotide-binding sites (Szopa, 1994; 1995). 
Here we show that the growth inhibition caused by induction of overexpression of the MBP in E. coli has been accompanied by apoptotic-like DNA fragmentation. When the expressed protein contained the complete 14-3-3 protein-derived polypeptide fused with MBP, both growth inhibition and DNA fragmentation were retarded. Expression of the polypeptide with deleted $\mathrm{N}$-terminus (protein kinase $C$ domain) caused rapid DNA degradation and immediate arrest of proliferation.

\section{Results}

The main purpose of the work presented here was to find out how the expression of 14-3-3-related polypeptide influences bacterial proliferation. The members of the 14-3-3 protein family display several activities, of which the regulation of protein kinase $\mathrm{C}$ (PKC) activity is the most consistent. To ascertain the significance of the PKC pseudosubstrate domain in the 14-3-3 protein we expressed the cDNA encoding the full size protein and its truncated version (PKC domain deleted) in the pMAL c2 vector in E. coli (see Figure 1).

\section{Proliferation of cells overexpressing 14-3-3 protein}

Bacteria transformed with the full size 14-3-3 cDNA and its truncated version $(\Delta \mathrm{N}-14-3-3)$ were grown at $37^{\circ} \mathrm{C}$. When $\mathrm{OD}_{600}$ reached 0.4 the bacteria were treated with $1 \mathrm{mM}$ IPTG. The aliquots of the bacteria corresponding to the equal optical density collected at different time-points after induction with IPTG were plated on YT/Amp solid medium. After incubation

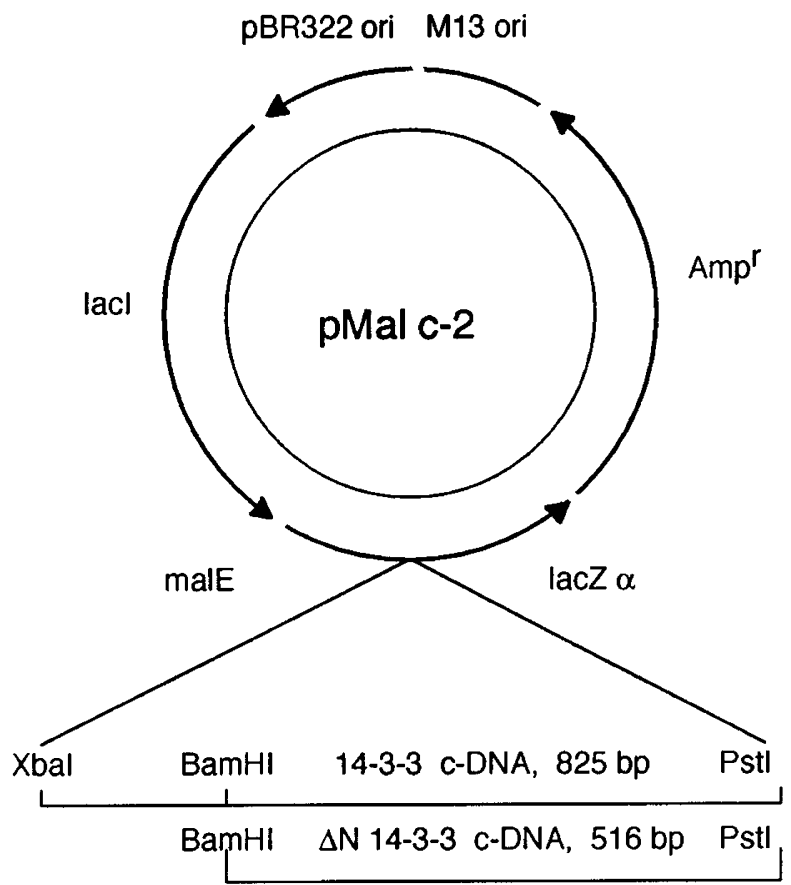

Figure 1 Schematic representation of the plasmids used in this work Expression vector pMal c2. full-size version, and truncated version of the cDNA isolated from C. pepo, coding for the 14-3-3 protein. overnight the resulting colonies were counted. Figure 2 shows which fraction of the proliferating cells present in the noninduced culture produces colonies when extracted at various time-points after induction. Induction slows down the proliferation of the bacteria synthesizing the maltose binding protein coded by multi-copy plasmids (filled triangles) compared with bacteria without plasmid (empty triangles). Synthesis of the protein obtained by fusion of the 14-3-3 derived polypeptide with the maltose binding protein influenced the proliferation of the bacteria in different ways, depending on whether the protein contained the PKC domain (filled squares) or not (empty squares).

\section{Examination of the integrity of the bacterial chromosomal DNA}

The genomic DNA of the bacterial cells was analyzed by pulse field gel electrophoresis. Figure 3 shows that overexpression of the protein coded by the multicopy plasmid causes degradation of bacterial DNA (Figure 3 lanes 3, 4 and 5). At $18 \mathrm{~h}$ after induction of the plasmid-encoded protein the degradation was advanced in bacteria independently of the nature of the protein synthesized. The DNA from the bacteria collected at earlier time points show various extents of degradation depending on the nature of the insert. Fusion with 14-3-3 polipeptides can either slow down (lane 4) or accelerate (lane 5) the DNA degradation observed in the case of the synthesis of the MBP (lane 3).

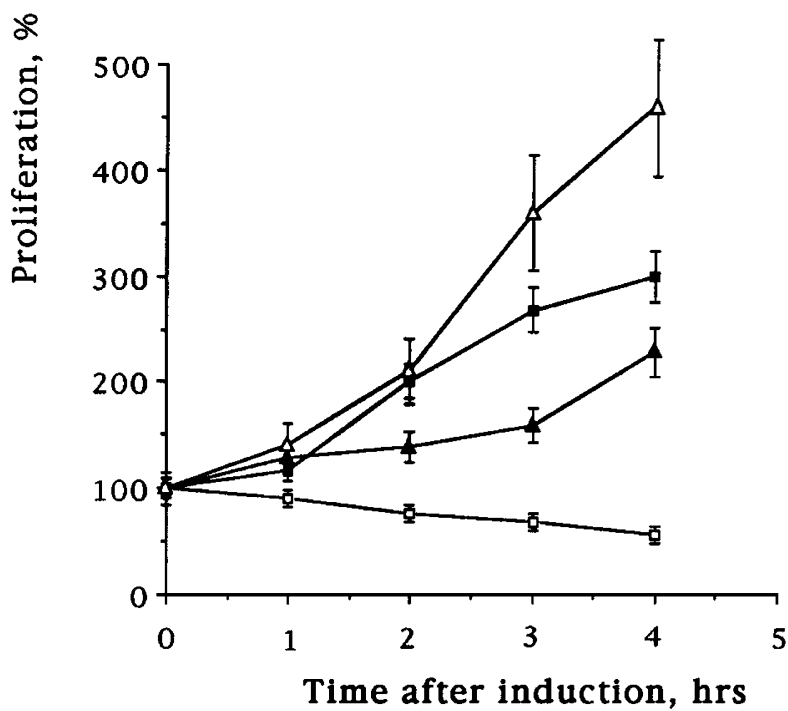

Figure 2 Proliferation of $E$. coli cells after induction of synthesis of plasmidencoded protein. Three $\mathrm{ml}$ of the medium was inoculated with the $50 \mu \mathrm{l}$ overnight culture. Three hours after inoculation the cells were stimulated by addition of IPTG to final concentration $1 \mathrm{mM}$. Serial dilutions of the aliquots corresponding to successive time-points were prepared from the stimulated culture and were plated on the agar. The number of colonies obtained from unstimulated cultures was used as a reference (100\%). Empty triangles: no plasmid, filled triangles: plasmid expressing MBP protein, filled squares: plasmid expressing MBP protein fused to the full-size 14-3-3 polypeptide, empty squares: the construct contained 14-3-3 polypeptide with PKC box deleted. The experiment has been performed in triplicate. The vertical bars show the standard deviations. 


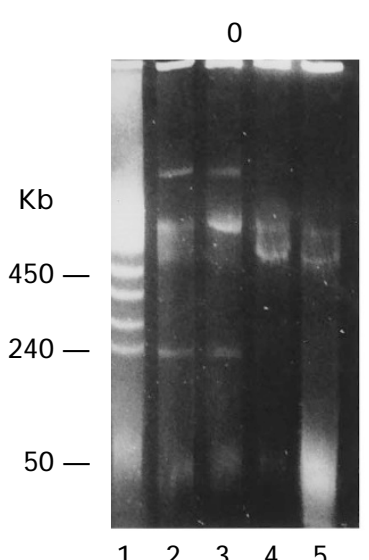

$\begin{array}{lllll}1 & 2 & 3 & 4 & 5\end{array}$

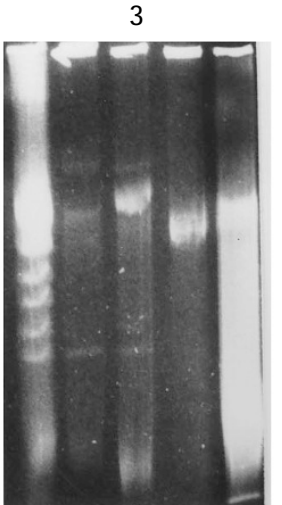

$\begin{array}{lllll}1 & 2 & 3 & 4 & 5\end{array}$

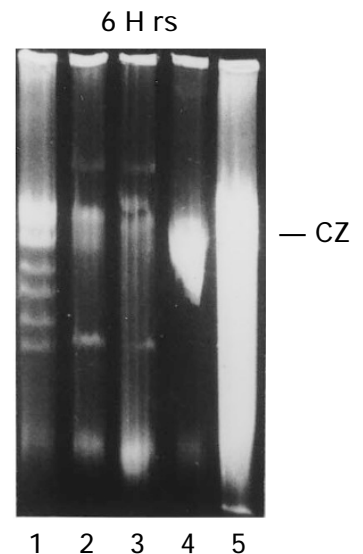

15

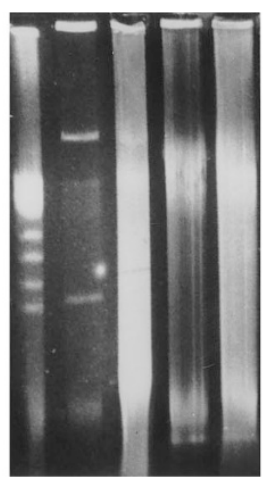

$\begin{array}{lllll}1 & 2 & 3 & 4 & 5\end{array}$

$18 \mathrm{H} \mathrm{rs}$

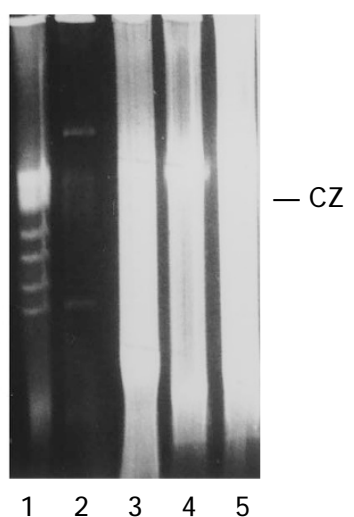

Figure 3 Pulsed field agarose gel electrophoresis of E. coli DNA extracted from transformed bacterial cells after stimulation. Lane 1: MW standard; lane 2: nontransformed bacteria; lane 3: vector with no insert; lane 4: p-Mal-cDNA ${ }^{14-3-3}$; lane 5: $p-M a l-c D N A^{\Delta N 14-3-3}$. The successive panels correspond to DNA extracted at six different time points following stimulation as indicated.

\section{Synthesis of the recombinant 14-3-3 protein in bacterial cells}

The bacterial cells collected at different time points after induction were analyzed for recombinant protein synthesis (Figure 4). Although the bacteria contained the same amount of plasmid DNA and the plasmids contained the expected 825 and $516 \mathrm{bp}$ long inserts for the full length and truncated protein respectively we wanted to make sure that they produced the expected protein in equivalent quantities. We found that the induction of synthesis of the protein coded by the plasmids caused the appearance of the expected 42,73 and $61.5 \mathrm{kDa}$ heavy bands on the Coomassie-blue stained gel. These bands corresponded to the maltose binding protein, full-size recombinant protein and the truncated version of the protein, respectively. Immunoblotting using antibody directed against the recombinant protein (not shown) confirmed their identity.

\section{Discussion}

Induction of the overexpression of the maltose-binding protein encoded by multicopy plasmid slows down the proliferation of the E. coli host cells. We have found that this slowing down is accompanied by the fragmentation of the bacterial chromosomal DNA into high molecular weight products. Similar fragmentation accompanies apoptosis in mammalian cells (Filipski, 1990). When the maltose binding protein was fused with the full size 14-3-3 nuclear protein from $C$. pepo the slowing down of the proliferation was delayed. The fragmentation of the DNA caused by overexpression of this gratuitous protein was also retarded for several hours. On the other hand, overexpression of the truncated version of the fusion protein caused immediate arrest of bacterial proliferation and rapid fragmentation of the bacterial chromosomal DNA (Figure 2 and 3 ).

The most important conclusion of this work is that arrest of bacterial cell proliferation caused by overexpression of the gratuitous proteins studied here is always accompanied by fragmentation of the chromosomal DNA. Although we cannot tell whether this fragmentation is the prime cause of the bacterial cell death, it is certainly an early symptom. We conclude also that even closely related proteins may show dramatic differences as far as their effect on the cell proliferation and DNA integrity is concerned. It suggests that, at least in the case of the 


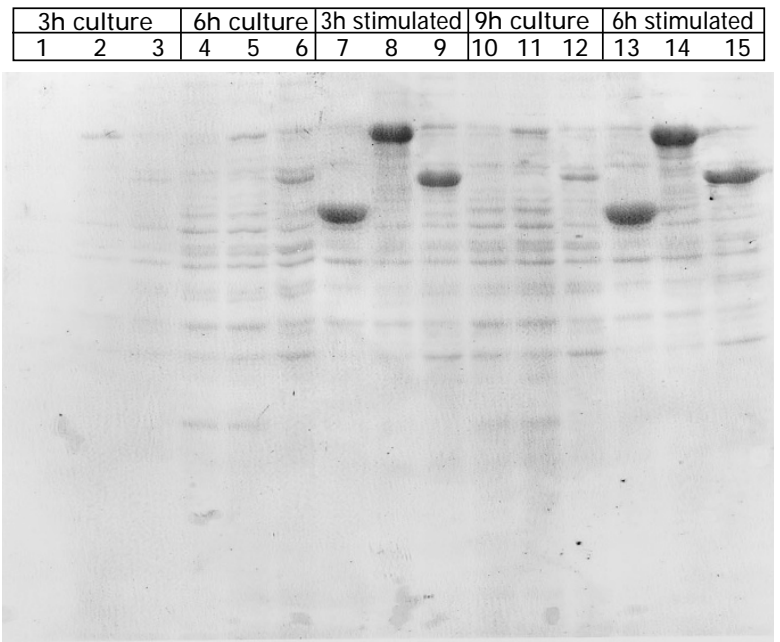

Figure 4 SDS polyacrylamide gel electrophoresis of bacterial protein. The cells were transformed with the $p$-Mal vector alone (lanes 1, 4, 7, 10 and 13) the vector containing full-size insert (lanes 2, 5, 8, 11 and 14) or the deleted form of the insert (lanes 3, 6, 9, 12 and 15). Three $\mathrm{ml} \mathrm{YT/Amp} \mathrm{medium} \mathrm{was}$ inoculated with $20 \mu \mathrm{l}$ of the overnight culture, and, after $3 \mathrm{~h}$ of growth (lanes 1 3 ), was supplemented (lanes $7-9$ and 13-15) with $1 \mathrm{mM} \mathrm{IPTG.} \mathrm{The} \mathrm{aliquots}$ were harvested 3 and $6 \mathrm{~h}$ after the stimulation. The extract from $5 \times 10^{6}$ cells was loaded onto each lane. The $12 \%$ polyacrylamide gel was stained with Coomassie blue after electrophoresis.

derivatives of the maltose-binding proteins studied here, starvation caused by 'protein-burden' does not explain the deleterious effect of the overexpression. The nature of the overexpressed protein is of the utmost importance for the process of initiation of cell death. The influence of the 143-3 protein isolated from $C$. pepo on this process is consistent with the idea that this protein may also be involved in the apoptosis in plants.

\section{Materials and Methods}

\section{Expression of 14-3-3 protein in bacteria and preparation of anti 14-3-3 antiserum}

The cDNA fragment encoding either the full size or truncated (first 103 aminoacids from $\mathrm{N}$-end deleted) 14-3-3 protein was amplified using two 32-nucleotide oligomers complementary to the $5^{\prime}$ - and $3^{\prime}$-end of the cDNA. The $5^{\prime}$-end and the $3^{\prime}$-end of the oligomers were constructed with Xbal and Pstl restriction sites, respectively. PCR amplification using 35 cycles of $94^{\circ} \mathrm{C}, 1 \mathrm{~min} ; 72^{\circ} \mathrm{C}, 2 \mathrm{~min}$. The amplified 14-3-3 cDNA was cleaved with $\mathrm{Xbal} / \mathrm{Pstl}$ and inserted into $\mathrm{Xbal} / \mathrm{Pstl}$ restriction site of pMal-c2. E. coli strain $\mathrm{XL}-1$ was transformed with the resulting construct and grown to OD 0.4 at $600 \mathrm{~nm}$ and at $37^{\circ} \mathrm{C}$. Synthesis of recombinant 14-3-3 protein was then induced by $1 \mathrm{mM}$ isopropyl- $\beta$-D-thiogalactopyranoside (IPTG) and the culture was grown for an additional time as specified. Cells were collected and lysed and the extract applied to a maltose-resin column (Bio-Rad). 14-3-3 protein purification by affinity chromatography was performed as described in the BioRad protocol. The recombinant 14-3-3 protein fused to the maltose binding protein was used for antiserum production in rabbit.

\section{SDS-PAGE and protein immunoblotting}

Solubilized protein was run on $12 \%$ SDS polyacrylamide gels and blotted electrophoretically onto nitrocellulose membranes (Schleicher and Schuell). Following transfer the membrane was sequentially incubated with blocking buffer ( $5 \%$ dry milk) and with the antibody directed against the 14-3-3 recombinant protein (1:1000 dilution). Formation and detection of the immune complex were performed as previously described (Szopa and Rose, 1986). Peroxidase-conjugated goat and anti-rabbit IgG served as a second antibody and were used at a dilution of 1:1500. 4-chloro-1-naphtol stain was used at a concentration of $0.6 \mathrm{mg} / \mathrm{ml}$.

\section{Pulsed field gel electrophoresis}

Bacterial cells were mixed with $2 \%$ low-melting point agarose, the blocks were treated with $0.05 \%$ proteinase $\mathrm{K}$ and washed with $50 \mathrm{mM}$ Tris $\mathrm{HCl}$, pH 7.0 containing 50 mM EDTA. Gels were run using CHEFDR2 pulse field electrophoresis apparatus (Bio-Rad) in 1\% agarose at $6 \mathrm{~V} / \mathrm{cm}$ and $20 \mathrm{sec}$ pulse time optimal for the separation of DNA fragments between 50 and $750 \mathrm{~kb}$.

\section{Acknowledgements}

This work was supported in part by KBN grant no 6P04AO4310 to JS, by grant no 520634 from the GREG and grant from ACC SV3 to JF, and by 'Projet Concerté de Cooperation Scientifique entre la France et la Pologne' (no 5089). EM's stay at the Institut J. Monod was financed by 'Jumelage Franco-Polonaise'.

\section{References}

Aitken A, Colligne DB, van Heuseden BPH, Isobe T, Roseboom PH, Rosenfeld G and Soll J (1992) 14-3-3 proteins: a highly conserved, widespread family of eucaryotic proteins. TIBS 17: 498-501

Aitken A (1995) 14-3-3 proteins on the MAP. TIBS 20: 95-97

Burbelo PD and Hall A (1990) Hot number in signal transduction. Curr. Biol. 5: 95-96

Conklin DS, Galakionov K and Beach D (1995) 14-3-3 protein associate with cdc 25 phosphatase. Proc. Natl. Acad. Sci. USA 92: 7892-7896

Dong H, Nilsson L and Kurland CG (1995) Gratuitous overexpression of genes in Escherichia colileads to growth inhibition and ribosome destruction. J. Bacteriol. 177: $1497-1504$

Filipski J, Leblanc J, Youdale T, Sikorska Mand Walker PR (1990) Periodicity of DNA folding in higher order chromatin structures. The EMBO J. 9: 1319-1327

Fu H, Xia K, Pallas DC, Cui C, Conroy K, Narishimhan RP, Mamon H, Collier RJ and Roberts TM (1994) Interaction of the protein kinase Raf-1 with 14-3-3 proteins. Science 266: 126-129

Hall BG (1990) Spontaneous point mutations that occur more often when advantageous than when neutral. Genetics 126: 5-16

Kurland CG and Dong H (1996) Bacterial growth inhibition by overproduction of protein. Mol. Microbiol. 21: 1-4

Morrison D (1994) 14-3-3: modulators or signaling proteins. Science 266: 56-57

Reuter GW, Fu H, Cripe LD, Collier RJ and Rendergast AM (1994) Association of the protein kinases C-Bcr and Bcr-Abl with proteins of 14-3-3 family. Science 266: $129-133$

Szopa J (1994) Cloning of a cDNA encoding a $32 \mathrm{kDa}$ nuclear matrix endonuclease from Cucurbita pepo which is highly similar to a family of mammalian neurotransmitter activators. J Plant Physiol 144: 617-619

Szopa J (1995) Expression analysis of a Cucurbita cDNA encoding endonuclease. Acta Biochim. Polon. 42: 183-190

Szopa J and Rose KM (1986) Cleavage of the 190-kDa subunit of DNA-dependent RNA polymerase yields small polypeptides capable of degrading DNA. J. Biol. Chem. 21: 9022-9028

Yarmolinsky MB (1995) Programmed cell death in bacterial populations. Science 267: $836-837$ 